\title{
Trends of Entamoeba histolytica Infections in a Tertiary Care Hospital of South India - A Three Year Perspective Study
}

\section{Gunalan Anitha, Mugunthan M, Nonika Rajkumari* and Gopika Jayan \\ Department of Microbiology, JIPMER, Puducherry, India \\ *Corresponding Author: Dr Nonika Rajkumari, Associate Professor, Department of Microbiology, Institute block, JIPMER, Dhanvantri Nagar, Puducherry, India.}

E-mail: nonika.raj@gmail.com
Received: August 10, 2020

Published: September 16, 2020

(C) All rights are reserved by Nonika

Rajkumari., et al.

\begin{abstract}
Background: Amoebiasis is still a leading cause of intestinal parasitic infections in the world. It can be asymptomatic or manifest a wide variety of presentation and hence its diagnosis of the pathogenic from the non-pathogenic is quite challenging.

Methodology: However, a changing pattern has been observed over the years with this pathogen. This study tried to explore the changing trends in patients infected with this pathogen over a period of 3 years.

Results: E. histolytica microscopy was positive in 55 cases with no gender predisposition. Most of the patients presented with mild abdominal pain associated with diarrhea. In this study we found that there is a decrease in prevalence of infection caused by various Entamoeba species. The overall positivity of infection was 3\% during the study period, which could most probably be due to improper sanitation favouring the transmission of the disease.

Conclusion: There has been increasing trend towards the non-pathogenic ones being recovered from the patients. This observation is especially applicable in light of the fact that expanding significance has been given to generally nonpathogenic species such as $E$. dispar and E. moshkovskii, since invasive amoebiasis has been exhibited to be related with these species. All things considered, the determinants of invasive amoebiasis are complicated and furthermore include different host factors.
\end{abstract}

Keywords: Amoebiasis; Microscopy; Trends; Entamoeba histolytica

\section{Introduction}

Amoebiasis is caused by pseudopod-forming, non-flagellated protozoan parasite Entamoeba histolytica. It is considered globally as a leading parasitic cause of human mortality and morbidity [13]. The disease manifestation is predominantly seen in developing countries affecting both the young and the old, malnourished individuals and pregnant women [4,5]. It very well may be asymptomatic or can prompt the advancement of amoebic liver ulcer, and amoebic colitis which is an unusual complication, however is related with high mortality, and on normal more than 50\% [6]. Furthermore, E. histolytica is one of the most widely recognized reasons for diarrhoea among travelers coming back from endemic areas [7]. National Institute of Allergy and Infectious Diseases (NIAID) has characterized E. histolytica as a class B priority biodefense organism due to its minimal infectious dose, ability to show resistance to chlorine, and natural steadiness which can represent a danger of simple dispersal through pollution of food and water supplies [8].

The diagnosis and conclusion of E. histolytica infection is difficult, and current techniques lack sensitivity. In most of the developing countries, diagnosis of Entamoeba infection is made by microscopic examination which is usually subjective with low sensitivity and specificity and also associated with high false positive 
outcomes owing from the nearness of E. dispar or E. moshkovskii. In this manner more explicit and more sensitive analytic methods have been created which incorporate enzyme immunoassays (ELISA) for the quick discovery of the adhesin of E. histolytica in human fecal samples and Polymerase Chain Reaction (PCR)- that permits to separate between E. histolytica and E. dispar infection which prompted a re-evaluation of the illness burden $[9,10]$.

Molecular test might be valuable as an extra to microscopic examination and can recognize pathogenic and non-pathogenic organism [11-13]. E. histolytica usually shows its most noteworthy tops in the wet season and step by step diminishes with the appearance of dry season. In an examination in Bangladesh, it was indicated that wet condition isn't the main factor that influences the detection curve of E. histolytica, however anti -carbohydrate recognition Domain IgA level in the gut is another deciding component for its event in that population [4]. In any case, E. histolytica infection usually follows a specific seasonality and pattern. The purpose of this study is to evaluate the factors influencing the occurrence of Entamoeba histolytica infection.

\section{Materials and Methods}

Our study was a cross sectional study which was carried out in a tertiary care hospital in south India from July, 2016 to June, 2019. Stool samples were collected from 1795 patients from various departments like medicine, surgery, paediatrics, pulmonary medicine, gynecology etc. suspected of having infection and the sample was screened microscopically for Entamoebae using saline wet mount and Lugol's iodine and was further subjected to formalin-ether concentration technique for visualization of cyst and trophozoite for Entamoeba [19].

\section{Results}

The whole study was carried out from July -2016 to June 2019. Total study population during the period of three years was found to be 1795 (Table 1). E. histolytica microscopy was positive in 55 cases. Male female ratio is 1:1 and there was no gender predisposition. Most of the positive cases (20 cases) were from medicine wards. Of the total positives, 12 cases were from pediatric age group. It was also observed that 42 out of 55 positive cases were from rural setup. Most of the patients presented with mild abdominal pain associated with diarrhea. All the positive samples have cyst forms with 5 samples showing trophozoite forms as well. The seasonal distribution was shown in figure 1 . However, no seasonality was observed in our study.

\begin{tabular}{|l|c|c|}
\hline Year & Total sample & $\begin{array}{c}\text { Positive by stool } \\
\text { microscopy }\end{array}$ \\
\hline 2016( July - Dec) & 275 & 13 \\
\hline 2017( Jan - Dec) & 650 & 31 \\
\hline 2018 (Jan - Dec) & 621 & 10 \\
\hline 2019 (Jan - June) & 249 & 1 \\
\hline
\end{tabular}

Table 1: Distribution of samples and positivity of Entamoeba spp. per year.

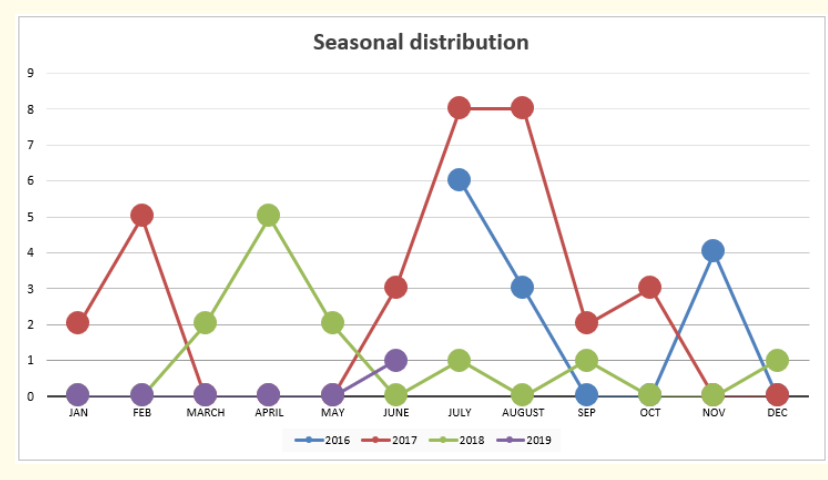

Figure 1: Seasonal distribution of Entamoeba histolytica.

The population studied is situated in a sociodemographic and ecological settings which is portrayed by deficiencies in proper cleaning. However, over the time period of our study, it slowly improved from $50 \%$ open defecation to building and using closed toilets in their homes. This is more so in cases of patients coming from rural areas. All together our finding shows that there in decreasing prevalence of infection caused by Entamoeba complex.

\section{Discussion and Conclusion}

The current study is done to estimate the frequency, epidemiology and other related components of Entamoeba histolytica infections. We performed a survey in a tertiary care hospital in south India from July 2016 to June 2019 by procuring the socio-demographic data from these patients. Stool samples were collected and microscopic examination was done. The overall positivity of infection was $3 \%$ during the study period, which could most probably be due to improper sanitation favouring the transmission of the disease.

Amoebiasis, a disease by protozoa E. histolytica is evaluated as the third driving parasitic reason for human mortality after malaria 
and schistosomiasis, making 40,000 to 100000 deaths yearly [15]. The worldwide significance of amoebiasis is across the board, with the most noteworthy weight of amoebiasis borne by those living in developing nations, especially the tropics and subtropics, where there is lack cleanliness and access to proper sanitation [5]. Numerous individuals are infected with E. histolytica, making amebic colitis as a main cause of diarrohea. Amoebiasis is endemic in developing countries of central and South America, Africa, and Asia. In developing nations, the burden of amoebiasis is hard to measure, and reports can be influenced by geographic locale, study configuration, test size, manifestation seriousness, and the sensitivity of the demonstrative methodology used [5]. A key issue for understanding the morbidity related with amoebiasis is to characterize the extent of diseases related with the pathogenic species $E$. histolytica. Strikingly, concentrates in various districts have indicated that numerous subjects infected with indistinguishable $E$. histolytica/E. dispar/E. moshkovskii complex and E. hartmanni parasites really harbor low-pathogenicity species such as E. dispar, E. moshkovskii, or even E. hartmannii [16]. In any case, this distinction requires detailed examination of the nuclear structures, which requires preparation of permanent smear, a visual micrometer and an exceptionally skilled parasitologist. These standards are difficult to meet for some labs [12]. The same findings were also observed in our study where microscopy fails to distinguish between them. Subsequently, routine parasitological strategies are not appropriate for separating these organisms. This impediment can prompt a critical number of patients being treated with antiparasitic medications, for example, metronidazole, who are not really be infected with E. histolytica. This perception is especially applicable in light of the fact that expanding significance has been given to customarily nonpathogenic species such as E. dispar and E. moshkovskii, since invasive amoebiasis has been exhibited to be related with these species [14]. Almost certainly, the determinants of invasive amoebiasis are complicated and furthermore include different host factors.

These asymptomatic infection found in our study gave off an impression that most of them are caused by transcendently nonpathogenic species or parasites with low pathogenic potential, however E. histolytica was likewise observed. In an urban quarter of Dhaka, Bangladesh, 11\% of children had amebic loose stools in the primary year of life [5]. By cross-sectional study, E. histolytica was identified in $13.7 \%$ of fecal samples in upper east states of In- dia [5]. According to the reports from other tropical nations, particularly in Bangladesh, which is topographically nearest to Kolkata, E. histolytica as a rule shows its most noteworthy peak in the wet season and step by step diminishes with appearance of dry season [20]. No seasonality was observed in our study (Figure 1).

The renaming of E. histolytica into Entamoeba complex including pathogenic E. histolytica and nonpathogenic E. dispar and E. moshkovskii has additionally added to the multifaceted nature of amoebiasis finding and epidemiology [17]. Fecal microscopy, the most commonly utilized clinical method for a long time; especially in asset restricted settings can't separate these three species with the exception of in uncommon invasive situations, where fecal examples much of the time found to contain hematophagous trophozoites. It was evaluated that on a normal just $1 \%$ of all out $E$. histolytica diseases develop into obtrusive structure and rest stay asymptomatic [15]. In like manner, stool culture based demonstrative strategies are time consuming, difficult and regularly unrewarding, with sensitivity of just about half. Other than microscopy and stool culture, ELISA based technique are among the different other approaches followed for explicit identification and location of E. histolytica in fecal samples. Few studies have however shown that, direct examination of the faecal samples have poor sensitivity and specificity due to cross contaminations with different parasites [11].

Studies from various geological zones of the world showed that the force of intestinal parasitic diseases (IPIs) including E. histolytica was fundamentally higher among children [18]. This is in contrast to our findings where majority of the positive cases were from adults. However, our outcomes didn't show any critical distinction in the predominance of E. histolytica disease when looked between gender. Our study findings were in line with other studies from different parts of the globe [5]. In contrast, most emergency clinic based examinations revealed sexual orientation subordinate E. histolytica infection [2]. Geographical location and country foundation of patients was additionally connected with E. histolytica disease, as appeared by different past examinations [4]. Our investigation further affirmed a higher danger of E. histolytica disease among the rural population, where prevailing poverty, no introduction to wellbeing instruction program, poor financial status, low norms of sanitation and cleanliness are the related components that added to the high pace of infection. 
We tried to evaluate the socio-demographic and parasitic profile of $E$. histolytica cases attending our tertiary care hospital. Many cases were from rural setup. Our overall positivity rate was around $3 \%$ which is lesser than other parts of the country. The decreasing trend is seen in the number of cases since last year. These could be due to increased awareness about the spread of this infection or a combination of methods is needed for it. The exact burden of infection can be calculated by incorporating antigen detection and molecular assays.

\section{Bibliography}

1. Gunther J., et al. "Amebiasis-related mortality among United States residents, 1990-2007". American Journal of Tropical Medicine and Hygiene 85.6 (2011): 1038-1040.

2. Montague BT., et al. "The immunosuppressed patient". Infection Disease: A Geo Gui (2011): 374.

3. Alavi KA. "Amebiasis". Clinical Colorectal Cancer Surgery 20 (2007): 33-37.

4. Haque R., et al. "Epidemiologic and clinical characteristics of acute diarrhea with emphasis on Entamoeba histolytica infections in preschool children in an urban slum of Dhaka, Bangladesh". American Journal of Tropical Medicine and Hygiene 69.4 (2003): 398-405.

5. Shirley DA., et al. "A review of the global burden, new diagnostics, and current therapeutics for amebiasis". Open Forum Infectious Diseases 5.7 (2018): 161.

6. Kantor M., et al. "Entamoeba histolytica: Updates in Clinical Manifestation, Pathogenesis, and Vaccine Development". Canadian Journal of Gastroenterology and Hepatology (2018): 4601420.

7. Nowak P., et al. "Entamoeba histolytica-pathogenic protozoan of the large intestine in humans". Journal of Clinical Microbiology and Biochemical Technology 1.1 (2015): 010-017.

8. Greene JM., et al. "National Institute of Allergy and Infectious Diseases bioinformatics resource centers: new assets for pathogen informatics". Infection Immunology 75.7 (2007): 3212-3219.

9. Khan U., et al. "Detection of Entamoeba histolytica using polymerase chain reaction in pus samples from amebic liver abscess". Indian Journal of Gastroenterology 25.2 (2006): 55.

10. Singh A., et al. "Rapid diagnosis of intestinal parasitic protozoa, with a focus on Entamoeba histolytica". Interdisciplinary Perspectives on Infectious Diseases (2009).

11. Fotedar R., et al. "Laboratory diagnostic techniques for Entamoeba species”. Clinical Microbiology Reviews 20.3 (2007): 511-532.
12. Blessmann J., et al. "Real-time PCR for detection and differentiation of Entamoeba histolytica and Entamoeba dispar in fecal samples". Journal of Clinical Microbiology 40.12 (2002): 44134417.

13. Orosz E., et al. "Real-time PCR assay for rapid qualitative and quantitative detection of Entamoeba histolytica". Acta Microbiologica Etimmunologica Hungarica 59.4 (2012): 451-460.

14. Khairnar K., et al. "Diagnosis of intestinal amoebiasis by using nested polymerase chain reaction-restriction fragment length polymorphism assay". Journal of Gastroenterology 42.8 (2001): 631-640.

15. Nath J., et al. "Molecular Epidemiology of Amoebiasis: A CrossSectional Study among North East Indian Population". PLOS Neglected Tropical Diseases 9.21 (2015): e0004225.

16. Calegar D., et al. "Frequency and molecular characterisation of Entamoeba histolytica, Entamoeba dispar, Entamoeba moshkovskii, and Entamoeba hartmanni in the context of water scarcity in northeastern Brazil". Memórias do Instituto Oswaldo Cruz 111.2 (2016): 114-119.

17. Shnawa B. "Molecular Diagnosis of Entamoeba histolytica, Entamoeba dispar, and Entamoeba moshkovskii: An Update Review". Annual Research and Review in Biology 21.5 (2017): $1-12$.

18. Sitotaw B., et al. "Prevalence of intestinal parasitic infections and associated risk factors among Jawi primary school children, Jawi town, north-west Ethiopia". BMC Infection Disease 19.1 (2019): 341.

19. Sastry A., et al. "Essentials of medical Parasitology". $2^{\text {nd }}$ Edition (2018).

20. Mukherjee AK., et al. "Trend of Entamoeba histolytica infestation in Kolkata". Gut Pathogens 2.1 (2010): 12.

\section{Assets from publication with us}

- Prompt Acknowledgement after receiving the article

- Thorough Double blinded peer review

- Rapid Publication

- Issue of Publication Certificate

- High visibility of your Published work

Website: https://www.actascientific.com/

Submit Article: https://www.actascientific.com/submission.php Email us: editor@actascientific.com

Contact us: +919182824667 